\title{
Obscured Asymptotic Giant Branch Stars in the Magellanic Clouds
}

\author{
Jacco Th. van Loon \\ Astronomical Institute, University of Amsterdam, Kruislaan 403, \\ NL-1098 SJ Amsterdam, The Netherlands
}

\begin{abstract}
.
I present results from an ongoing study of the evolution and mass loss of AGB stars with optically thick circumstellar envelopes in the Magellanic Clouds.
\end{abstract}

\section{Introduction}

Asymptotic Giant Branch (AGB) stars are highly evolved stars with intermediate progenitor masses and can be studied in great detail in the Magellanic Clouds, avoiding disadvantages in the Milky Way such as unknown distances or severe interstellar extinction. Because of the differences in metallicity between these galaxies, several aspects of stellar evolution can be studied for their metallicity dependence. Alternatively, the presence and properties of an AGB population in a galaxy reflect on the metallicity and star formation history in the past $10^{8-10} \mathrm{yr}$.

The most important phenomena that (can) occur to a star when it ascends the AGB are Long Period Variability (LPV), the transformation into a carbon star, and mass loss at rates up to $10^{-5} \mathrm{M}_{\odot} \mathrm{yr}^{-1}$ or more. The stars with the highest mass-loss rates are obscured from the optical by their dust shells, but can still be bright at infrared wavelengths. We have searched for AGB counterparts of IRAS point sources in the direction of the Magellanic Clouds. Carbon stars had not been found at luminosities higher than $M_{\text {bol }} \sim-6$ mag in optical surveys (e.g., Iben 1981; Hughes \& Wood 1990; Costa \& Frogel 1996), but they might be found among obscured AGB stars.

Obscured AGB stars have very red infrared colors, typically $(H-K) \gtrsim 1$ mag. The near-infrared color depends on the mass-loss rate, but also on the dust-to-gas ratio and the luminosity, and on the expansion velocity $v_{\text {exp }}$ of the shell. We can measure $v_{\text {exp }}$ from circumstellar maser emission profiles. These velocities are also an excellent means for determining the kinematics and mass distributions in the Magellanic Clouds.

\section{Results}

We successfully searched for near-infrared AGB stars as counterparts of IRAS point sources, mainly in the LMC (Loup et al. 1997; Zijlstra et al. 1996; van Loon et al. 1997, 1998a). Together with already known obscured AGB stars and 
the recent search in the SMC by Groenewegen \& Blommaert (1998), the samples of obscured AGB stars consist now of $\sim 50$ stars in the LMC and $\sim 25$ in the SMC. In the LMC there are $\sim 3$ times as many IRAS sources left unexplored. IRAS picked up sources with $S_{25 \mu} \geq 0.1 \mathrm{Jy}$. With ISO we were able to study bolometrically fainter or less obscured stars with $S_{25 \mu} \gtrsim 0.01 \mathrm{Jy}$.

Using color characteristics and infrared spectroscopy from the ground (ESO, SAAO, CTIO) and space (IRAS, ISO), we established the chemical composition for many of the obscured AGB stars in the LMC. We found the most luminous carbon star in the Magellanic Clouds, IRAS04496-6958 with $M_{\text {bol }}=-6.8 \mathrm{mag}$, and another candidate carbon star with $M_{\text {bol }} \sim-7.0 \mathrm{mag}$. Thus carbon stars do exist up to the AGB tip luminosity. Due to mass loss the stellar mantle is reduced, thereby switching off "Hot Bottom Burning" that otherwise prevents massive stars from becoming carbon stars.

The optical depth of the circumstellar dust shell increases with mass-loss rate, but also with decreasing luminosity of the underlying star. Very obscured AGB stars exist in both the LMC and the SMC, despite their low metallicities. Assuming a dust-to-gas ratio proportional to the metallicity, the near-infrared colors of the LMC and SMC stars suggest very similar mass-loss rates. However, the outflow velocities in the shell are expected to decrease with decreasing metallicity, causing an increase of the optical depth. Hence SMC stars may in fact have lower mass-loss rates than LMC stars.

Expansion velocities based on measurements of $\mathrm{OH}$ profiles alone are not very reliable, as is shown by our recent $\mathrm{SiO}$ and $\mathrm{H}_{2} \mathrm{O}$ maser detections (van Loon et al. 1996, 1998b) of known OH masers in the LMC (Wood et al. 1992). We argue that the expansion velocities may have been generally underestimated, and more maser searches need to be performed in the Magellanic Clouds before conclusions can be drawn on the metallicity dependence of the expansion velocity and the mass-loss rate.

Acknowledgments. I wish to thank the organizers for a pleasant and interesting conference and for a travel grant. $\mathrm{E}$ para o anjinho Joana: muito obrigado, por tudo.

\section{References}

Costa, E., \& Frogel, J.A. 1996, AJ, 112, 2607

Groenewegen, M.A.T., \& Blommaert, J.A.D.L. 1998, A\&A, 332, 25

Hughes, S.M.G., \& Wood, P.R. 1990, AJ, 99, 784

Iben, I. 1981, in Physical Processes in Red Giants, I. Iben Jr. \& A. Renzini, Dordrecht: Reidel, 3

Loup, C., et al. 1997, A\&AS, 125, 419

van Loon, J.Th., et al. 1996, A\&A, 306, L29

van Loon, J.Th., et al. 1997, A\&A, 325, 585

van Loon, J.Th., et al. 1998a, A\&A, 329, 169

van Loon, J.Th., et al. 1998b, A\&A, 337, 141

Wood, P.R., et al. 1992, ApJ, 397, 552

Zijlstra, A.A., et al. 1996, MNRAS, 279, 32 